\title{
ALETAMENTO MATERNO E A PRÁTICA DE DESMAME EM DUAS COMUNIDADES RURAIS DE PIRACICABA - SP1
}

\section{BREAST FEDING AND WEANING IN TWO RLRAL COMMUNIIES OF A BRAZUAN TOWN (PIRACICABA-SP)}

\author{
Kátia Cilene TABAI ${ }^{2}$ \\ J osé Ferreira de CARVALHO ${ }^{3}$ \\ Elisa bete SALAY4
}

\begin{abstract}
RESUMO
O aleitamento materno e a prática de desmame foram estudados em 76 crianças de 3 a 24 meses de idade pertencentes a 69 famílias de comunidades rurais de Piracicaba, SP, por meio de entrevistas. Foram analisadas as medianas da duração do aleitamento materno exclusivo, predominante (incluindo somente água e chá) e misto, utilizando-se a tábua de vida. O teste Wilcoxon foi aplicado para verificar as variáveis socioeconômicas e demográficas associadas com o tempo de aleitamento. A mediana da duração de aleitamento materno exclusivo foi de 3 dias para Anhumas e 30 dias para Santa Olímpia. O aleitamento predominante e o aleitamento misto prolongaram-se por 90 e 180 dias em Anhumas e 120 e 165 dias em Santa Olímpia, respectivamente. Somente o sexo da criança apresentou associação com a duração do aleitamento materno. Iniciou-se o processo de desmame especialmente com água, chás, leite de vaca fluido e sucos açucarados.
\end{abstract}

Termos de indexação: aleitamento materno, alimentos infantis, desmame, população rural, Brasil.

\begin{abstract}
Breast feeding and weaning were studied in 76 infants from 3 to 24 months of age in two rural communities in Piracicaba, state of São Paulo. Interviews with the children's mothers were conducted at their homes. The average duration of exclusive breast feeding, predominant breast feeding (including only water and tea) and complementary feeding was analyzed by means of life tables. The Wilcoxon test was conducted to verify socioeconomic and demographic variables associated with the duration of breast feeding. The average
\end{abstract}

\footnotetext{
(1) Trabalho elaborado a partir da dissertação de mestrado em Ciência da Nutrição de Kátia Cilene TABAI: A prática da alimentação de crianças (3 a 24 meses de idade) das famílias de bairros rurais de Piracicaba-SP. Campinas, 1997, pela Faculdade de Engenharia de Alimentos da UNICAMP.

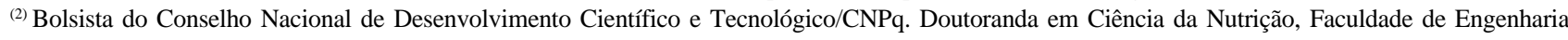
de Alimentos/Departamento de Planejamento Alimentar e Nutrição da UNICAMP.

(3) Estastítico da Statistika Consultoria, Pesquisador do Núcleo de Informática Biomédica da UNICAMP.

(4) Professora do Departamento de Planejamento Alimentar e Nutrição da Faculdade de Engenharia de Alimentos da UNICAMP, a quem toda correspondência deve ser endereçada, DEPA - FEA - UNICAMP, Caixa Postal 6121, 13083-970, Campinas, SP, Brasil, E-mail: salay@fea.unicamp.br
} 
duration of exclusive breast feeding was 3 days in the community of Anhumas and 30 days in that of Santa Olímpia. Predominant breast feeding and complementary feeding were prolonged for 90 and 180 days in Anhumas and for 120 and 165 days in Santa Olímpia, respectively. Only the sex of the child was associated with the duration of predominant breast feeding, with boys being nursed longer. Insufficient milk was the main reason given for termination of breast feeding. Weaning was generally initiated with liquids such as water, tea, fluid cow milk and sweetened juices.

\section{INTRO DUÇÃO}

Index terms: breast feeding, infant food, weaning, rural population, Brazil.
Prolongar o aleitamento materno exclusivo até os seis primeiros meses de vida das crianças ainda é um objetivo não alcançado no país. Na verdade, a Pesquisa Nacional sobre Saúde e Nutrição (PNSN), realizada em 1989 no Brasil, mostrou que a maioria das crianças é desmamada completamente antes do quarto mês de vida (INSTITUTO..., 1992). O mesmo estudo confirmou por intermédio de uma análise comparativa com a situação observada em 1974/1975 que apesar dos esforços desenvolvidos no país nesta última década, os índices de desmame ${ }^{5}$ precoce ainda são elevados. Após o nascimento $97 \%$ das crianças são amamentadas ao seio, mas logo no terceiro mês de vida, 43\% delas são desmamadas (INSTITUTO..., 1990).

Outras pesquisas realizadas no Brasil, em sua maioria com populações de áreas menores, revelaram que a duração da amamentação exclusiva é curta (VILLA \& PELÁ et al., 1989; COSTA et al., 1993; ASSIS et al., 1994; BARROS et al., 1994; SIQUEIRA et al., 1994). O término precoce do aleitamento materno e, conseqüentemente, a introdução de alimentos complementares antes do período recomendado, tem ocorrido também nos países desenvolvidos(LOUGHLIN etal., 1985; FEINSTEIN et al., 1986; KOKINOS \& DEWEY, 1986; O’QUINN et al., 1991; SISKIND et al., 1993).

Diversos estudos analisaram os fatores associados com a duração do aleitamento materno em países menos desenvolvidos (ELLIOT et al., 1985; FEINSTEIN et al., 1986; ISSLER et al., 1989; BARRIA et al., 1990; COSTA et al., 1993; ASSIS et al., 1994; IZURIETA \& LARSON, 1995). Segundo FORMAN (1984) destacam-se os fatores socioeconômicos, características da mãe da criança, fatores clínicos, orientações e atitudes sobre planejamento familiar.

O aleitamento materno oferece ótimas condições para o desenvolvimento das crianças, como édescrito amplamente pela literatura. O conhecimento do período de amamentação das crianças, assim como os primeiros alimentos complementares utilizados e as variáveis socioeconômicas determinantes destas práticas, é relevante para a saúde pública, principalmente no caso de países em desenvolvimento, onde grande parte da população não tem acesso a condições de vida satisfatórias.

Os estudos na zona rural do Brasil sobre o aleitamento materno são escassos (ASSIS et al., 1994; PRADO et al, 1995). Esta pesquisa objetivou então analisar a amamentação, a duração do período de aleitamento materno e a respectiva prática de desmame. Foram estudadas todas as crianças no período de desmame ( 3 a 24 meses de idade) de famílias dos bairros rurais Anhumas e Santa Olímpia, no município de Piracicaba. Os fatores socioeconômicos e demográficos que podem interferir nessas práticas foram também analisados.

\section{MATERIAL E MÉTODOS}

\section{Local da pesquisa e população analisada}

Piracicaba é um município do Estado de São Paulo, localizado na Região Administrativa de Campinas, possui $1452 \mathrm{~km}^{2}$ de extensão territorial (144 km² de zona urbana e $1308 \mathrm{~km}^{2}$ de zona rural). A população de Piracicaba, observada no último censo, foi de 283,9 mil habitantes, dividindo-se em cerca de 270 mil pessoas na zona urbana e 13,9 mil habitantes na zona rural (INSTITUTO..., 1991). Em 1993, a população foi calculada em pelo menos 350 mil habitantes (FUNDAÇÃO..., 1993).

Foram selecionados os bairros Anhumas e Santa Olímpia, no município de Piracicaba, por suas características de zonas rurais e por serem habitados por famílias de diferentes níveis socioeconômicos. Desses bairros rurais, estudou-se então a totalidade

\footnotetext{
${ }^{(5)}$ O período de desmame deve ser compreendido como a introdução de outro alimento além do materno, que vai desde a introdução do novo alimento, inclusive chás e sucos, até a suspensão completa da amamentação, segundo o Comitê Nacional da Atenção Alimentar e Nutricional (INSTITUTO..., s.d.).
} 
das crianças com idade entre 3 e 24 meses de idade, a saber, 55 crianças pertencentes a 49 famílias do bairro Anhumas e 21 crianças de 20 famílias do bairro Santa Olímpia no período de 13 a 27 de janeiro de 1996.

\section{Instrumentos de medida}

Para a obtenção dos dados foram realizadas entrevistas domiciliares, utilizando-se questionários previamente testados. Este instrumento continha questões sobre características socioeconômicas e demográficas da família, aleitamento materno e práticas de desmame. Foram utilizadas as definições de aleitamento materno exclusivo, aleitamento predominante (incluindo somente água e chá) e aleitamento materno misto, relatadas pela Organização Mundial de Saúde (WORLD..., 1991) e pelo INSTITUTO... (s.d.), a saber:

- aleitamento materno exclusivo: o recém-nascido só recebe o leite materno e nenhum outro líquido ou alimento sólido, com exceção de gotas ou xaropes de vitaminas, remédios ou sais minerais.

- aleitamento materno predominante: a principal fonte de alimentação da criança é o leite materno. Todavia, ela também pode receber água e chás.

- aleitamento materno misto: o leite materno é complementado com outros alimentos.

Quanto à recomendação do aleitamento materno, empregou-se a do extinto Instituto Nacional de Alimentação e Nutrição (INAN) ${ }^{6}$, ou seja, que o aleitamento materno se estenda, de forma exclusiva, durante os seis primeiros meses de vida das crianças (LEÃO et al., 1992).

\section{Processamento de dados e análise estatística}

A tabulação dos dados foi feita em um banco de dados desenvolvido especificamente para o projeto, usando o programa Microsoft Access versão 2.0. As medianas da duração do aleitamento materno exclusivo, predominante (incluindo água e chás) e misto, foram obtidas por meio da técnica estatística tábua de vida, usada amplamente na literatura pesquisada (MONTEIRO, 1988; INSTITUTO..., 1992; ASSIS et al., 1994). Realizou-se essa análise estatística pelo procedimento Lifetest, contido no SAS (SAS INSTITUTE, 1985).

A duração do aleitamento materno exclusivo, predominante e misto, foi associada com diferentes características socioeconômicas e demográficas das famílias. Utilizou-se para este procedimento o teste de Wilcoxon, contido no SAS.

\section{RESULTADOS}

A Tabela 1 apresenta a distribuição por faixa etária e por sexo das crianças. A proporção de crianças, na faixa etária de 12-24 meses foi de 58,2\% em Anhumas e 47,6\% em Santa Olímpia. Nas duas comunidades encontrou-se número maior de meninas: 58,2\% e 61,9\% em Anhumas e Santa Olímpia respectivamente.

Tabela 1. Faixa etária e sexo das crianças dos bairros rurais de Piracicaba - SP, 1996.

\begin{tabular}{|c|c|c|c|c|c|c|}
\hline \multirow{2}{*}{ Faixa etária e sexo } & \multicolumn{2}{|c|}{ Anhumas } & \multicolumn{2}{|c|}{ Santa Olímpia } & \multicolumn{2}{|c|}{ Total } \\
\hline & $\mathrm{n}$ & $\%$ & $\mathrm{n}$ & $\%$ & $\mathrm{n}$ & $\%$ \\
\hline \multicolumn{7}{|l|}{ Faixa etária } \\
\hline 3-5,9 meses & 7 & 12,7 & 2 & 9,5 & 9 & 11,1 \\
\hline 6-11,9 meses & 16 & 29,1 & 9 & 42,9 & 25 & 36,0 \\
\hline $12-24$ meses & 32 & 58,2 & 10 & 47,6 & 42 & 52,9 \\
\hline Total & 55 & 100,0 & 21 & 100,0 & 76 & 100,0 \\
\hline \multicolumn{7}{|l|}{ Sexo } \\
\hline Masculino & 23 & 41,8 & 8 & 38,1 & 31 & 39,9 \\
\hline Feminino & 32 & 58,2 & 13 & 61,9 & 45 & 60,1 \\
\hline Total & 55 & 100,0 & 21 & 100,0 & 76 & 100,0 \\
\hline
\end{tabular}

(6) Outros órgãos sugerem que o aleitamento materno exclusivo se estenda até os primeiros quatro a seis meses de vida, entre eles o Órgão Oficial da Sociedade Latinoamericana e o Comitê de Nutrição da Academia Americana de Pediatria (AMERICAN..., 1980; O’DONNELL, 1988). 
As características socioeconômicas e demográficas das famílias estão relacionadas na Tabela 2. Em ambos os grupos a maioria das mães tinha de 20 a 30 anos de idade, nasceram em Piracicaba; 100,0\% do bairro Santa Olímpia e 67,3\% das mães do bairro Anhumas. A renda mensal familiar per capita e a escolaridade das mães de Santa Olímpia foram superiores às encontradas no bairro Anhumas. A proporção de crianças que não moravam com o pai foi de $9,1 \%$ para Anhumas e 9,5\% para Santa Olímpia.

Das 69 famílias, 84,5\% das mães não trabalhavam. Em Anhumas 6,1\% das mães das crianças trabalhavam e em Santa Olímpia, 25,0\%. A religião predominante das mães em ambas as comunidades foi a católica, perfazendo o total de 63,3\% para Anhumas e 100,0\% para Santa Olímpia.

Nota-se que a maioria das crianças foi amamentada ao seio ao nascer (Anhumas, 89,1\% e Santa Olímpia, 90,5\%). No momento da realização da entrevista domiciliar, 30,9\% das crianças do bairro Anhumas e 19,1\% do bairro Santa Olímpia, ainda estavam sendo amamentadas. Em Anhumas, 56,4\% das mães tinham experiência em amamentação. No bairro Santa Olímpia, todavia, este índice foi de $28,6 \%$. Nos dois bairros a maioria das mães recebeu orientação sobre como alimentar a criança. A totalidade das mães de Santa Olímpia e 89,2\% daquelas de Anhumas obtiveram de médicos pediatras essas informações (Tabela 3).

As Figuras 1, 2 e 3, cuja elaboração foi utilizada a tábua de vida, mostram o tempo de aleitamento materno em cada caso. Considerando-se os três tipos de aleitamento materno estudados, nota-se que as crianças do bairro Santa Olímpia foram amamentadas por período maior do que as de Anhumas, exceção feita ao aleitamento misto. Todavia, em ambas as comunidades, a maior parte das crianças foi desmamada antes do período recomendado pelo Ministério da Saúde. A mediana da duração do aleitamento materno exclusivo foi de 3 dias para as crianças do bairro Anhumas e de 30 dias para as crianças do bairro Santa Olímpia. O tempo de aleitamento materno predominante foi de 90 dias para o bairro Anhumas e 120 dias para Santa Olímpia. Constatou--se que a mediana duração do aleitamento materno misto foi de
180 dias para Anhumas e 165 dias para Santa Olímpia.

Realizou-se o teste estatístico de associação da duração do aleitamento materno com as variáveis: sexo da criança, faixa etária da mãe da criança, escolaridade da mãe, renda familiar per capita, experiência anterior em amamentação, orientação sobre como alimentar a criança, vínculo empregatício da mãe e apoio do pai da criança (o pai mora com a criança). Não foi aplicado este teste para o local de criação e religião da mãe da criança, pois nas populações analisadas essas características tiveram pouca ou nenhuma variação (Tabela 2).

A aplicação do teste estatístico de Wilcoxon mostrou que, entre as variáveis socioeconômicas e demográficas analisadas, apenas o sexo da criança apresentou associação com efeito no tempo de aleitamento materno predominante (Tabela 4). O tempo de aleitamento materno predominante foi de 90 dias para as crianças do sexo masculino e de 60 dias para as do sexo feminino.

Apesar do tempo de aleitamento materno das crianças ter sido inferior às recomendações nas duas comunidades estudadas, observou-se que a maioria das mães considerava que a amamentação deveria se prolongar por mais de um ano (Tabela 5). Em ambos os bairros, o item mais citado como o principal motivo de ter deixado de amamentar foi o leite insuficiente (42,1\% das mães em Anhumas e 35,3\% em Santa Olímpia). No bairro Anhumas, outros fatores importantes foram: rejeição da criança ao peito $(21,1 \%)$; nova gravidez $(16,5 \%)$ e saúde da criança (10,5\%). Para o bairro Santa Olímpia o término do leite materno $(17,6 \%$ das mães) foi a razão mais relatada, após o leite insuficiente (Tabela 6).

As Tabelas 7 e 8 apresentam a idade em que foram introduzidos diferentes tipos de alimentos na dieta de crianças. Nas faixas etárias abaixo de 6 meses de idade observou-se que as crianças receberam diversos alimentos. Assim, nos dois bairros a maioria das crianças iniciou com menos de 2 meses de idade a ingestão de líquidos, como água e chá, e de açúcares. Elas consumiam também, mas em menor proporção, leite de vaca fluido, outros tipo de leite e sucos.

O consumo de cereais, legumes, frutas, 
Tabela 2. Características socioeconômicas e demográficas das famílias nos bairros de Piracicaba - SP, 1996.

\begin{tabular}{|c|c|c|c|c|c|c|}
\hline \multirow{2}{*}{ Características } & \multicolumn{2}{|c|}{ Anhumas } & \multicolumn{2}{|c|}{ Santa Olímpia } & \multicolumn{2}{|c|}{ Total } \\
\hline & $\mathrm{n}$ & $\%$ & $\mathrm{n}$ & $\%$ & $\mathrm{n}$ & $\%$ \\
\hline \multicolumn{7}{|l|}{ Faixa etária das mães } \\
\hline$<20$ anos & 12 & 24,5 & 1 & 5,0 & 13 & 14,7 \\
\hline 20 a 30 anos & 29 & 59,2 & 13 & 65,0 & 32 & 62,2 \\
\hline 31 a 40 anos & 7 & 14,3 & 6 & 30,0 & 13 & 22,1 \\
\hline+40 anos & 1 & 2,0 & 0 & 0,0 & 1 & 1,0 \\
\hline Total & 49 & 100,0 & 20 & 100,0 & 69 & 100,0 \\
\hline \multicolumn{7}{|l|}{ Escolaridade das mães } \\
\hline $1^{\circ} \mathrm{Grau}$ incompleto & 40 & 81,6 & 3 & 15,0 & 43 & 48,3 \\
\hline $1^{\circ}$ Grau completo & 6 & 12,3 & 3 & 15,0 & 9 & 13,6 \\
\hline $2^{\circ} \mathrm{Grau}$ completo & 3 & 6,1 & 13 & 65,0 & 16 & 35,6 \\
\hline Superior completo & 0 & 0,0 & 1 & 5,0 & 1 & 2,5 \\
\hline Total & 49 & 100,0 & 20 & 100,0 & 69 & 100,0 \\
\hline Local de nascimento das mães & & & & & 53 & \\
\hline Piracicaba & 33 & 67,3 & 20 & 100,0 & 8 & 83,7 \\
\hline Outros municípios de São Paulo & 8 & 16,3 & 0 & 0,0 & 4 & 8,1 \\
\hline Estado do Paraná & 4 & 8,2 & 0 & 0,0 & 4 & 4,1 \\
\hline Outros Estados & 4 & 8,2 & 0 & 0,0 & 69 & 4,1 \\
\hline Total & 49 & 100,0 & 20 & 100,0 & & 100,0 \\
\hline Renda familiar per capita & & & & & 27 & \\
\hline até $1 / 2$ salário mínimo (s.m.)* & 25 & 51,0 & 2 & 10,0 & 14 & 30,5 \\
\hline$+1 / 2$ a 1 s.m. & 13 & 26,6 & 1 & 5,0 & 20 & 15,8 \\
\hline+1 a 2 s.m. & 10 & 20,4 & 10 & 50,0 & 8 & 35,2 \\
\hline+2 s.m. & 1 & 2,0 & 7 & 35,0 & 69 & 18,5 \\
\hline Total & 49 & 100,0 & 20 & 100,0 & & 100,0 \\
\hline Vínculo empregatício das mães & & & & & 8 & \\
\hline Sim & 3 & 6,1 & 5 & 25,0 & 61 & 15,5 \\
\hline Não & 46 & 93,9 & 15 & 75,0 & 69 & 84,5 \\
\hline Total & 49 & 100,0 & 20 & 100,0 & & 100,0 \\
\hline Religião das mães & & & & & 51 & \\
\hline Católica & 31 & 63,2 & 20 & 100,0 & 9 & 81,6 \\
\hline Não católica & 9 & 18,4 & 0 & 0,0 & 9 & 9,2 \\
\hline Sem religião & 9 & 18,4 & 0 & 0,0 & 69 & 9,2 \\
\hline Total & 49 & 100,0 & 20 & 100,0 & & 100,0 \\
\hline \multicolumn{7}{|l|}{ Apoio do pai da criança** } \\
\hline Sim & 45 & 91,8 & 18 & 90,0 & 63 & 91,3 \\
\hline Não & 4 & 8,2 & 2 & 10,0 & 6 & 8,7 \\
\hline Total & 49 & 100,0 & 20 & 100,0 & 69 & 100,0 \\
\hline
\end{tabular}

* O valor do salário mínimo de janeiro de 1996 era de 100 reais.

** O pai mora com a criança. 
Tabela 3. Características do aleitamento materno e orientação sobre alimentação de crianças, em bairros rurais de Piracicaba-SP, 1996.

\begin{tabular}{|c|c|c|c|c|c|c|}
\hline \multirow{2}{*}{ Características } & \multicolumn{2}{|c|}{ Anhumas } & \multicolumn{2}{|c|}{ Santa Olímpia } & \multicolumn{2}{|c|}{ Total } \\
\hline & $\mathrm{n}$ & $\%$ & $\mathrm{n}$ & $\%$ & $\mathrm{n}$ & $\%$ \\
\hline \multicolumn{7}{|c|}{ Aleitamento materno } \\
\hline Sim & 32 & 58,2 & 15 & 71,4 & 47 & 64,8 \\
\hline Não & 6 & 10,9 & 2 & 9,5 & 8 & 10,2 \\
\hline Ainda amamenta & 17 & 30,9 & 4 & 19,1 & 21 & 25,0 \\
\hline Total & 55 & 100,0 & 21 & 100,0 & 76 & 100,0 \\
\hline \multicolumn{7}{|c|}{$\begin{array}{l}\text { Experiência anterior em } \\
\text { amamentação }\end{array}$} \\
\hline Sim & 31 & 56,4 & 6 & 28,6 & 37 & 42,5 \\
\hline Não & 24 & 43,6 & 15 & 71,4 & 39 & 57,5 \\
\hline Total & 55 & 100,0 & 21 & 100,0 & 76 & 100,0 \\
\hline \multicolumn{7}{|c|}{$\begin{array}{l}\text { Orientação da mãe sobre } \\
\text { alimentação }\end{array}$} \\
\hline Sim & 28 & 50,9 & 16 & 76,2 & 44 & 63,6 \\
\hline Não & 27 & 49,1 & 5 & 23,8 & 32 & 36,4 \\
\hline Total & 55 & 100,0 & 21 & 100,0 & 76 & 100,0 \\
\hline \multicolumn{7}{|c|}{ Origem da orientação } \\
\hline Médicos pediatras & 25 & 89,2 & 16 & 100,0 & 41 & 93,1 \\
\hline Enfermeira & 1 & 3,6 & 0 & 0,0 & 1 & 2,3 \\
\hline Mãe/Parentes & 1 & 3,6 & 0 & 0,0 & 1 & 2,3 \\
\hline Assistente social & 1 & 3,6 & 0 & 0,0 & 1 & 2,3 \\
\hline Total & 28 & 100,0 & 16 & 100,0 & 44 & 100,0 \\
\hline
\end{tabular}

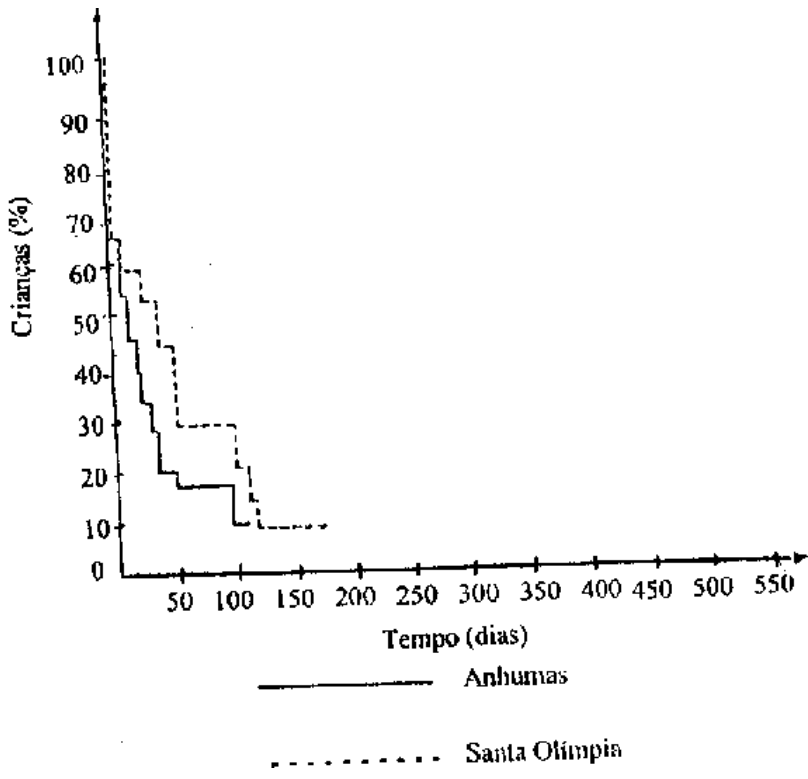

Figura 1. Tempo de aleitamento materno exclusivo das crianças de comunidades rurais, obtido em análise de sobrevivência em Piracicaba, 1996

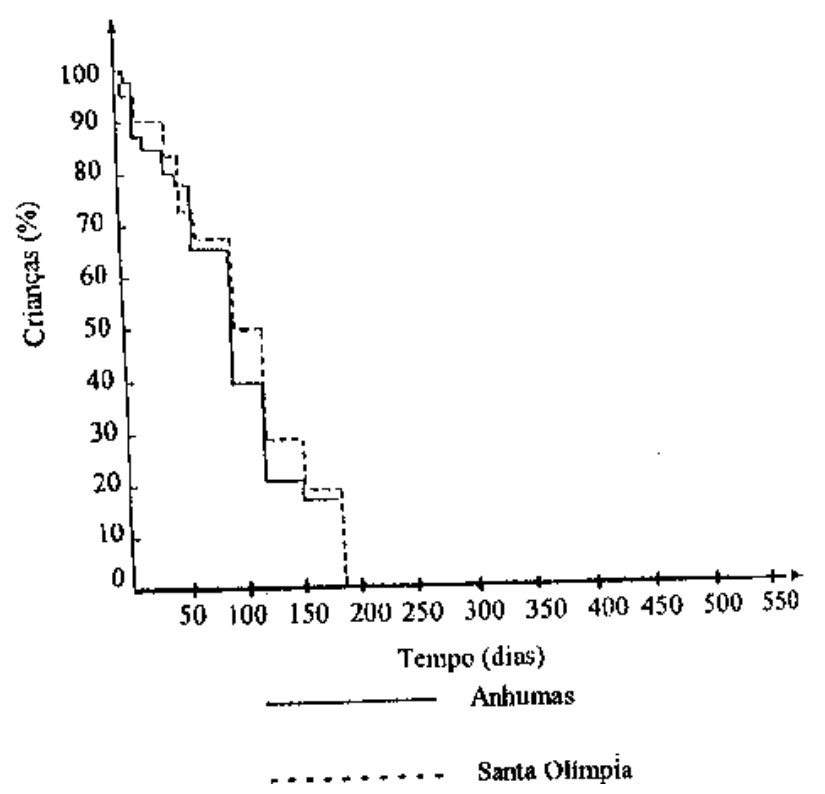

Figura 2. Tempo de aleitamento materno predominante(incluindo água e chá) das crianças de comunidades rurais, obtido em análise de sobrevivência em Piracicaba, 1996 

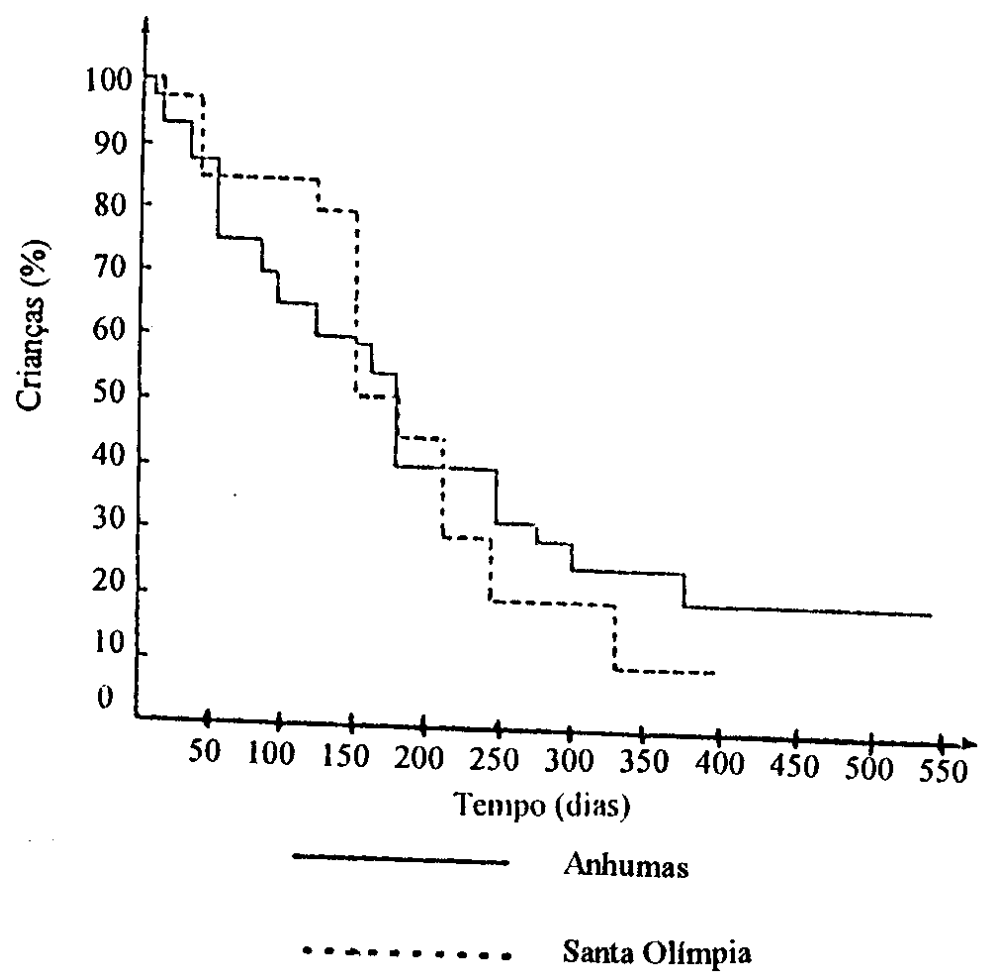

Figura 3. Tempo de aleitamento materno total das crianças de comunidades rurais, obtido em análise de sobrevivência em Piracicaba, 1996.

Tabela 4. Associação de tempo de aleitamento materno predominante com variáveis usando teste de Wilcoxon nas comunidades rurais de Piracicaba-SP, 1996.

\begin{tabular}{lcc}
\hline Variáveis & Teste & $\mathrm{P}$ \\
\hline Sexo da criança & $-8,7337$ & 0,0214 \\
Faixa etária da mãe da criança & $-0,7867$ & 0,8719 \\
Escolaridade da mãe da criança & 2,6114 & 0,5944 \\
Renda familiar per capita & $-0,3760$ & 0,9598 \\
Experiência em amamentação & $-4,5267$ & 0,2218 \\
Vínculo empregatício da mãe da criança & $-1,8896$ & 0,4622 \\
Orientação sobre alimentação & 4,8971 & 0,1868 \\
Apoio do pai da criança & 0,4338 & 0,8654 \\
\hline
\end{tabular}

Tabela 5. Tempo de amamentação considerado ideal pelas mães dos bairros rurais de Piracicaba-SP, 1996.

\begin{tabular}{|c|c|c|c|c|c|c|}
\hline \multirow{2}{*}{ Tempo de amamentação } & \multicolumn{2}{|c|}{ Anhumas } & \multicolumn{2}{|c|}{ Santa Olímpia } & \multicolumn{2}{|c|}{ Total } \\
\hline & $\mathrm{n}$ & $\%$ & $\mathrm{n}$ & $\%$ & $\mathrm{n}$ & $\%$ \\
\hline 0,5 - 4,0 meses & 3 & 6,1 & 1 & 5,0 & 4 & 5,6 \\
\hline $4,1-6,0$ meses & 11 & 22,5 & 4 & 20,0 & 15 & 21,2 \\
\hline $6,1-12$ meses & 6 & 12,2 & 7 & 35,0 & 13 & 23,6 \\
\hline mais de 12 meses & 29 & 59,2 & 8 & 40,0 & 37 & 49,6 \\
\hline Total & 49 & 100,0 & 20 & 100,0 & 69 & 100,0 \\
\hline
\end{tabular}


Tabela 6. Principal motivo alegado pelas mães por ter deixado de amamentar as crianças dos bairros rurais de Piracicaba-SP, 1996.

\begin{tabular}{|c|c|c|c|c|c|c|}
\hline \multirow{2}{*}{ Motivos } & \multicolumn{2}{|c|}{ Anhumas } & \multicolumn{2}{|c|}{ Santa Olímpia } & \multicolumn{2}{|c|}{ Total * } \\
\hline & $\mathrm{n}$ & $\%$ & $\mathrm{n}$ & $\%$ & $\mathrm{n}$ & $\%$ \\
\hline Leite insuficiente & 16 & 42,1 & 6 & 35,3 & 22 & 38,7 \\
\hline Rejeição da criança ao peito & 8 & 21,1 & 1 & 5,9 & 9 & 13,5 \\
\hline Nova gravidez & 4 & 10,5 & 0 & 0,0 & 4 & 5,2 \\
\hline Saúde da criança & 4 & 10,5 & 0 & 0,0 & 4 & 5,2 \\
\hline Trabalho da mãe da criança & 2 & 5,3 & 2 & 11,8 & 4 & 8,6 \\
\hline Outros motivos & 2 & 5,3 & 3 & 17,6 & 5 & 11,4 \\
\hline Término do leite materno & 1 & 2,6 & 3 & 17,6 & 4 & 10,1 \\
\hline Saúde da mãe da criança & 1 & 2,6 & 1 & 5,9 & 2 & 4,3 \\
\hline Falta de tempo para amamentar & 0 & 0,0 & 1 & 5,9 & 1 & 3,0 \\
\hline Total & 38 & 100,0 & 17 & 100,0 & 55 & 100,0 \\
\hline
\end{tabular}

* Na época da pesquisa 17 mães estavam amamentando em Anhumas e 4 mães em Santa Olímpia.

Tabela 7. Idade de introdução de alimentos na dieta de 55 crianças no bairro rural Anhumas em Piracicaba-SP, 1996.

\begin{tabular}{|c|c|c|c|c|c|c|c|c|c|c|}
\hline \multirow{2}{*}{ Alimentos } & \multicolumn{2}{|c|}{$0-1,9$ meses } & \multicolumn{2}{|c|}{$2-3,9$ meses } & \multicolumn{2}{|c|}{4 a 5,9 meses } & \multicolumn{2}{|c|}{6 a 12 meses } & \multicolumn{2}{|c|}{+12 meses } \\
\hline & $\mathrm{n}$ & $\%$ & $\mathrm{n}$ & $\%$ & $\mathrm{n}$ & $\%$ & $\mathrm{n}$ & $\%$ & $\mathrm{n}$ & $\%$ \\
\hline Água & 35 & 63,6 & 17 & 30,9 & 0 & 0,0 & 1 & 1,8 & 0 & 0,0 \\
\hline Chá & 41 & 74,5 & 5 & 9,1 & 1 & 1,8 & 1 & 1,8 & 0 & 0,0 \\
\hline Leite de vaca fluido & 8 & 14,5 & 14 & 25,4 & 9 & 16,4 & 10 & 1,8 & 1 & 1,8 \\
\hline Outros leites* & 5 & 9,1 & 3 & 5,4 & 1 & 1,8 & 3 & 5,4 & 0 & 0,0 \\
\hline Sucos naturais de frutas & 3 & 5,4 & 24 & 43,6 & 14 & 25,4 & 6 & 11,0 & 0 & 0,0 \\
\hline Cereais & 0 & 0,0 & 9 & 16,4 & 21 & 38,2 & 17 & 30,9 & 0 & 0,0 \\
\hline Legumes & 0 & 0,0 & 9 & 16,4 & 20 & 36,4 & 14 & 25,4 & 0 & 0,0 \\
\hline Frutas & 0 & 0,0 & 14 & 25,4 & 16 & 29,1 & 14 & 25,4 & 0 & 0,0 \\
\hline Verduras & 0 & 0,0 & 3 & 5,4 & 14 & 25,4 & 9 & 16,4 & 2 & 3,7 \\
\hline Carnes & 0 & 0,0 & 6 & 11,0 & 15 & 27,3 & 21 & 38,2 & 0 & 0,0 \\
\hline Ovos & 0 & 0,0 & 1 & 1,8 & 15 & 27,3 & 16 & 29,1 & 2 & 3,7 \\
\hline $\begin{array}{l}\text { Alimento industrializado tipo } \\
\text { "рара” }\end{array}$ & 0 & 0,0 & 0 & 0,0 & 5 & 9,1 & 1 & 1,8 & 0 & 0,0 \\
\hline Farinhas lácteas & 0 & 0,0 & 4 & 7,3 & 6 & 11,0 & 9 & 16,4 & 0 & 0,0 \\
\hline Refrigerantes $* *$ & 0 & 0,0 & 2 & 3,7 & 7 & 12,7 & 27 & 49,1 & 2 & 3,7 \\
\hline Açúcares & 30 & 54,5 & 13 & 23,6 & 3 & 5,4 & 1 & 1,8 & 0 & 0,0 \\
\hline Sal & 0 & 0,0 & 14 & 25,4 & 22 & 40,0 & 11 & 20,0 & 0 & 0,0 \\
\hline
\end{tabular}

* Outros leites diferentes do leite de vaca fluido, incluindo leite em pó.

** Bebida não alcoólica gaseificada. 
Tabela 8. Idade de introdução de alimentos na dieta de 21 crianças no bairro rural Santa Olímpia em Piracicaba-SP, 1996.

\begin{tabular}{|c|c|c|c|c|c|c|c|c|c|c|}
\hline \multirow[t]{2}{*}{ Alimentos } & \multicolumn{2}{|c|}{$0-1,9$ meses } & \multicolumn{2}{|c|}{2 - 3,9 meses } & \multicolumn{2}{|c|}{4 a 5,9 meses } & \multicolumn{2}{|c|}{6 a 12 meses } & \multicolumn{2}{|c|}{+12 meses } \\
\hline & $\mathrm{n}$ & $\%$ & $\mathrm{n}$ & $\%$ & $\mathrm{n}$ & $\%$ & $\mathrm{n}$ & $\%$ & $\mathrm{n}$ & $\%$ \\
\hline Água & 11 & 52,4 & 5 & 23,8 & 3 & 14,3 & 2 & 9,5 & 0 & 0,0 \\
\hline Chá & 13 & 61,9 & 2 & 9,5 & 1 & 4,7 & 0 & 0,0 & 0 & 0,0 \\
\hline Leite de vaca fluido & 5 & 23,8 & 1 & 4,8 & 6 & 28,6 & 4 & 19,0 & 0 & 0,0 \\
\hline Outros leites * & 2 & 9,5 & 2 & 9,5 & 2 & 9,5 & 2 & 9,5 & 0 & 0,0 \\
\hline Sucos naturais de frutas & 2 & 9,5 & 8 & 38,1 & 7 & 33,3 & 2 & 9,5 & 0 & 0,0 \\
\hline Cereais & 0 & 0,0 & 1 & 4,8 & 5 & 23,8 & 12 & 63,1 & 1 & 4,8 \\
\hline Legumes & 0 & 0,0 & 2 & 9,5 & 10 & 47,6 & 8 & 14,5 & 0 & 0,0 \\
\hline Frutas & 1 & 4,8 & 6 & 28,6 & 9 & 42,9 & 3 & 14,3 & 0 & 0,0 \\
\hline Verduras & 0 & 0,0 & 5 & 23,8 & 7 & 33,3 & 6 & 28,6 & 0 & 0,0 \\
\hline Carnes & 0 & 0,0 & 4 & 19,1 & 4 & 19,1 & 11 & 52,4 & 1 & 4,8 \\
\hline Ovos & 1 & 4,8 & 2 & 9,5 & 3 & 14,3 & 13 & 61,9 & 1 & 4,8 \\
\hline $\begin{array}{l}\text { Alimento industrializado tipo } \\
\text { "рара” }\end{array}$ & 0 & 0,0 & 0 & 0,0 & 0 & 0,0 & 0 & 0,0 & 0 & 0,0 \\
\hline Farinhas lácteas & 1 & 4,8 & 0 & 0,0 & 4 & 19,1 & 3 & 14,3 & 0 & 0,0 \\
\hline Refrigerantes ** & 1 & 4,8 & 0 & 0,0 & 2 & 9,5 & 14 & 66,7 & 2 & 9,5 \\
\hline Açúcares & 8 & 38,1 & 4 & 19,1 & 6 & 28,6 & 1 & 4,8 & 0 & 0,0 \\
\hline Sal & 1 & 4,8 & 3 & 14,3 & 6 & 28,6 & 9 & 42,9 & 1 & 4,8 \\
\hline
\end{tabular}

* Outros leites diferentes do leite de vaca fluido, incluindo leite em pó.

** Bebida não alcoólica gaseificada.

verduras, carnes, ovos e farinha láctea, pelas crianças, iniciou-se na faixa etária de 2 a 3,9 meses de idade, em ambos os bairros. Para as crianças com 4 a 5,9 meses de idade, os tipos de alimentos utilizados na dieta foram diversificados. Nota-se que, o leite de vaca fluido tinha sido introduzido para 56,3\% das crianças, na faixa etária abaixo de 6 meses, no bairro Anhumas e para 57,2\% no bairro Santa Olímpia. Dentre os alimentos industrializados analisados, destacou-se o consumo de refrigerantes nos dois bairros. O alimento industrializado tipo "papa” nunca foi consumido pela totalidade das crianças do bairro Santa Olímpia e pela maioria das de Anhumas. Menos de um quarto das crianças consumiam as farinhas lácteas antes de 6 meses de idade, em ambos os bairros.

\section{DISCUSSÃO E CONCLUSÃO}

Esta pesquisa verificou que o aleitamento materno se prolonga por tempo insuficiente nos bairros rurais de Piracicaba. As medianas da duração do aleitamento materno exclusivo, predominante e misto foram de 3, 90 e 180 dias, respectivamente, para as crianças do bairro Anhumas. Na comunidade de Santa Olímpia, esses indicadores alcançaram valores de 30, 120 e 165 dias.

Na pesquisa nacional de 1989, PNSN, constatou-se que na Região Sudeste rural a mediana do aleitamento materno total se estendia por 134 dias e o aleitamento materno exclusivo por 82 dias (LEÃO et al., 1992). Para um mesmo tipo de aleitamento materno, portanto, a duração encontrada para os bairros rurais do município de Piracicaba foi levemente superior a média da zona rural de 1989. Todavia, o tempo de aleitamento materno não atingiu às recomendações do Ministério da Saúde. Ressalta-se que análises feitas em zonas urbanas brasileiras encontraram tempo de aleitamento materno exclusivo superior aos obtidos neste estudo. Tendência oposta foi observada para o tempo de aleitamento total (MONTEIRO, 1988).

Apesar da duração do aleitamento materno ter sido inferior às recomendações, na opinião da maioria das mães, a amamentação deveria durar até mais de um ano de idade., Entretanto, as mães alegaram que deixaram de amamentar, principalmente por 
acreditarem ter pouco leite. Este motivo é também muito citado em outras pesquisas, na sua maioria, realizadas em zonas urbanas (FEINSTEIN et al., 1986; ISSLER et al., 1989; VILLA \& PELÁ, 1989; NOVOTNY et al., 1995;). Seria interessante, esclarecer se a crença de que o leite materno é insuficiente no Brasil esta ligada, por exemplo, a falta de informação, para depois, conceber-se intervenções (FEINSTEIN et al., 1986; VILLA \& PELÁ, 1989).

Nesta pesquisa somente o sexo da criança apresentou associação com o tempo de aleitamento predominante que foi maior para crianças do sexo masculino. Os costumes e tradições das populações dos bairros rurais, podem ter ocasionado essa diferença. Em uma população pobre da área urbana de Pelotas, no sul do Brasil, MARTINES et al. (1989) encontraram também associação com a duração do aleitamento materno e o sexo da criança. Neste caso, todavia a duração foi maior para as crianças do sexo feminino. Na opinião dos autores isto poderia ter ocorrido porque, as mães acreditam que as necessidades nutricionais das crianças do sexo masculino são maiores. Portanto, elas iniciam o processo de introdução de alimentação complementar antes do tempo devido. Nota-se ainda que em outros estudos foi constatada associação da duração do aleitamento materno com as diferentes variáveis: local de criação da mãe, escolaridade dos pais, idade da mãe da criança, mães multigestas e com experiência anterior em amamentação e renda familiar (ISSLER et al., 1989; VILLA \& PELÁ, 1989).

Este estudo mostrou ainda que, as bebidas açucaradas, como chás, foram introduzidas na dieta de grande parte dos lactentes, antes dos dois meses de idade. Este dado se assemelha aos de outras pesquisas do Brasil e portanto, podemos concluir que não se trata de uma tendência local (ASSIS et al., 1994; PRADO et al., 1995). Em ambos os bairros, outros alimentos utilizados no processo de desmame para a maioria das crianças foram o leite de vaca fluido, sucos de frutas naturais, legumes e frutas. Dosprodutos industrializados analisados, nenhum foi consumido por parte significativa das crianças, com exceção dos refrigerantes. Entretanto, outros estudos mostraram que, no Brasil, alimentos industrializados, como o leite em pó, têm sido freqüentemente consumidos no período de desmame, inclusive no meio rural (PRADO et al., 1995). Na verdade, o processo de aquisição de alimentos por uma população depende de fatores diversos como econômicos, sociais, culturais, sensoriais, entre outros (MARSHALL, 1995).

Os resultados desta pesquisa sugerem ainda que medidas eficazes de promoção do aleitamento materno exclusivo nos seis primeiros meses de vida das crianças devam ser implementadas. Programas educativos através dos meios de comunicação, nas rotinas hospitalares e em especial nas ações de base comunitárias, são exemplos de intervenções quepodem ser executadas de maneira eficaz (GIUGLIANI, 1994).

\section{REFERÊNCIAS BIBLIOGRÁFICAS}

AMERICAN ACADEMY OF PEDIATRICS. Committee on Nutrition. On the Feeding of Supplemental Foods to Infants Committee on Nutrition. Pediatrics, New York, v.65, n.6,p.1178-1181, 1980.

ASSIS, A.M.O., PRADO, M.S., FREITAS, M.C., SILVA, R.C., RAMOS, L.B., MACHADO, A.D. Prática do aleitamento materno em comunidades rurais do semiárido baiano. Revista de Saúde Pública, São Paulo, v.28,n.5,p.380-384, 1994.

BARRIA, M.C.M., OROZCO, E.U.B., GATICA, M.E.U.M., MACKENNEY, J.P., VALVERDE, C.G., DRAGO, M.T., VALENCIA, C.G. Introducción precoz de fórmulas lácteas en la alimentación del niño. Revista Chilena de Pediatria, Santiago de Chile, v.61, n.4, p.218-222, 1990 .

BARROS, F.C., HALPEM, R., VICTORA, C.G., TEIXEIRA, A.M.B., BÉRIA, J.U. Promoção da amamentação em localidade urbana da região sul do Brasil: estudo de intervenção randomizado. Revista de Saúde Pública, São Paulo v.28, n.4, p.277-283, 1994.

COSTA, M.C.O., FIGUEIREDO, E.M., SILVA, S.B. Aleitamento materno: causas de desmame ejustificativa para amamentar. Jornal de Pediatria, Rio de Janeiro, v.69, n.3,p. 176-178, 1993.

ELLIOTT, T.C., AGUNDA, K.O., KIGONDU, J.G., KINOTTI, S.N., LATHAM, M.C. Breastfeeding versus infant formula: the Kenyan case. Food Policy, Guildford,v.10,n.1, p.7-10, 1985.

FEINSTEIN, J.M., BERKELHMAER, J.E., GRUSZKA, M.E., WONG, C.A., CAREY, A.E. Factors related to early termination of breast-feeding in an urban population. Pediatrics, New York, v.78, n.2, p. 210-215, 1986 
FORMAN, M.R. Review of research on the factors associated with choice and duration of infant feeding in less-developed countries. Pediatrics, New York, v.74, n.4pt.2,p. 667-694, 1984.

FUNDAÇÃO SISTEMA ESTADUAL DE ANÁLISE DE DADOS. Perfil municipal 1980-1991: Região administrativa de Campinas. São Paulo, 1993. 49p.

GIUGLIANI, E.R.J. Amamentação: como e por que promover. Jornal de Pediatria, Rio de Janeiro, v.70, n.3,p.138-151,1994.

INSTITUTO BRASILEIRO DE GEOGRAFIA ESTATÍSTICA. Censo demográfico 1991: resultados do universo relativos às características da população e dos domicílios. São Paulo, 1991, p.75.(Número 21)

INSTITUTO NACIONAL DE ALIMENTAÇÃO E NUTRIÇÃO. Comitê Nacional de Atenção Alimentare Nutrição. Orientação alimentar e nutricional para o desmame: um período crítico do crescimento, Brasília : Ministério da Saúde, s.d. 20p. (Mimeografado).

INSTITUTO NACIONAL DE ALIMENTAÇÃO E NUTRIÇÃO. Pesquisa nacional sobre saúde $e$ nutrição (PNSN) 1989. Brasília, 1990. 43p. (Arquivo dos dados da Pesquisa).

INSTITUTO NACIONAL DE ALIMENTAÇÃO E NUTRIÇÃO. Pesquisa nacional sobre saúde e nutrição (PNSN):resultadospreliminares. 3.ed.Brasília, 1992.33p.

ISSLER, H., LEONE, C., QUINTAL, V.S. Duração do aleitamento materno em uma área urbana de São Paulo, Brasil. Boletin de la Oficina Sanitaria Panamericana, Washington DC, v.106, n.6, p.513-522, 1989.

IZURIETA, L.M., LARSON-BROWN, L. Child feeding practices in Guatemala. Ecology of Food and Nutrition, New York, v.33, p.249-262, 1995.

KOKINOS, M., DEWEY, K.G. Infant feeding practices of migrant mexican-american families in northern California. Ecology of Food and Nutrition, New York, v.18,n.3, p.209-220, 1986.

LEÃO, M.M., COITINHO, D.C., RECINE, E., COSTA, L.A.L., LACERDA, A.J. O perfil do aleitamento materno no Brasil. In: FUNDAÇÃO INSTITUTO BRASILEIRO DE GEOGRAFIA E ESTATÍSTICA (FIBGE). Perfil estatístico de crianças e mães no Brasil: aspectos de saúde e nutrição de crianças no Brasil 1989. Rio de Janeiro, 1992.p.97-110.

LOUGHLIN, H.H., CLAPP-CHANNING, N.E., GEHLBACH, S.H., POLLARD, J.C., McCUTCHEN, T.M. Early termination of breast-feeding: identifying those at risk. Pediatrics, New York, v.75, n.3, p. 508-513,1985.

MARSHALL, D.W. Food choice and the consumer. London : Blackie Academic \& Professional, 1995. 332p.
MARTINES, J.C., ASHWORTH, A., KIRKWOOD, B. Breast-feeding among the urban poor in southern Brazil: reasons for termination in the first 6 months of life. Bulletin of the World Health Organization, New York, v.67,n.2,p.151-161, 1989.

MONTEIRO, C.A. Saúde e nutrição das crianças de São Paulo: diagnóstico, contrastes sociais e tendências. São Paulo: Hucitec, 1988. 165 p.

NOVOTNY, R., KIEFFER,E., MOR, J., THIELE, M. Nativity and infant feeding patterns among filipino women in Hawaii. Ecology of Food and Nutrition, New York, v.33,p. 263-272, 1995.

O’DONNELL, A.M. Alimentación del niño en America Latina. Archivos Latinoamericanos de Nutrición, Guatemala, v.38, n.3, p.685-704, 1988. (Centro de Estudios sobre Nutrición infantil).

O’QUINN, J., McINTYRE, L., MEADE, S. Breast-feeding patterns of montserratian women. Bulletin of the Pan American Health Organization, Washington DC, v.25, n.4,p.320-325, 1991.

PRADO, M.S., ASSIS, A.M.O., FREITAS, M.C.S., SILVA, R.C.R., VARJÃO, M.L. Padrão e seleção de alimentos complementares e sucedâneos do leite materno em comunidades rurais no semi-árido baiano. Revista de Nutrição da Puccamp, Campinas, v.8, n.1, p.31-46, 1995.

SAS INSTITUTE. SAS user's guide: statistics. New York, 1985.956p.

SIQUEIRA, R., DURSO, N., ALMADA, A.G.P., MOREIRA, M.T., MASSAD, G. B. Reflexões sobre as causas do desmame precoce observadas em dinâmicas de grupo de incentivo ao aleitamento materno. Jornal de Pediatria, Rio de Janeiro, v.70, n.1, p.16-20, 1994.

SISKIND, V., MAR, C.D., BCHIR, M.B., SCHOFIELD, F. Infant feeding in Queensland Australia: long-term trends. American Journal of Public Health, Washington DC, v.83, n.1,p.103-106, 1993.

VILLA, T.C.S., PELÁ, N.T.R. Aleitamento materno e suplementação alimentar. Boletin de la Oficina Sanitaria Panamericana, Washington DC, v.106, n.2,p.108-16, 1989.

WORLD HEALTH ORGANIZATION. Indicators for assessing breast-feeding practices. Geneva, 1991.15p. (Report of an Informal Meeting 11-12 June).

Recebido para publicação em 10 de novembro de 1997 e aceito em 20 de março de 1998. 